SCIENTIFIC REPORT

\title{
Clinicopathological findings of choroidal neovascularisation following verteporfin photodynamic therapy
}

\author{
F Gelisken, B A Lafaut, W Inhoffen, M Voelker, S Grisanti, K U Bartz-Schmidt
}

Br J Ophthalmol 2004;88:207-211. doi: n10.1136/bjo.2003.018754

\begin{abstract}
Aims: To report the clinicopathologic findings of surgically excised choroidal neovascularisation (CNV) three days after verteporfin photodynamic therapy (PDT).

Methods: In three patients (three eyes) with age related macular degeneration, the CNV was surgically removed three days after PDT. The CNV specimens were examined by light microscopy.

Results: The patients had subfoveal classic CNV. Fluorescein angiography revealed non-perfusion of the CNV after PDT and before surgery in all eyes. The light microscopy of the CNV membranes showed swollen and damaged endothelium. Thrombus formation or vascular occlusion in the CNV vessels was not detected.

Conclusion: PDT did not cause a thrombosis of the vessels within the CNV three days after PDT. Severe endothelial damage of the CNV was observed and is likely a primary effect of PDT. Non-perfusion of the CNV at this stage is possibly secondary to occlusion at a deeper level, namely the underlying feeding choroid.
\end{abstract}

n the Western world, age related macular degeneration (AMD) is the leading cause of legal blindness in the elderly. ${ }^{1}$ The neovascular form of AMD leads to irreversible damage of the neurosensory retina resulting in severe loss of visual acuity. ${ }^{2}$

Verteporfin (Visudyne, Novartis AG, Buelach, Switzerland) photodynamic therapy (PDT) leads to photothrombosis of choroidal neovascularisation (CNV) without detectable damage of overlying neurosensory retina. ${ }^{3}$ Several clinical trials reported the beneficial effect of PDT in preventing visual loss in subfoveal neovascular $\mathrm{AMD} .^{45}$ Photodynamic therapy is based on photochemical reactions, generated by light irradiation of the photosensitiser. ${ }^{6}$ Formation of the singlet oxygen causes photo-oxidative damage to target tissue, namely CNV. Endothelial cell damage, resulting in vascular occlusion and blood flow stasis, is generally believed to be the principal mechanism of PDT.

Histopathological findings of CNV two weeks to three months after PDT in neovascular AMD have been published. ${ }^{7-}$ ${ }^{9}$ In these studies, endothelial damage of the CNV was noted. In a recent study, a CNV membrane secondary to presumed ocular histoplasmosis was extracted three days following the second PDT and showed partial occlusion of the CNV vessels. $^{10}$

Even though PDT is generally accepted as a means of treatment for neovascular AMD, limited information is available about the morphological changes of CNV shortly after such therapy. We herein report on clinicopathological findings of surgically excised CNV secondary to AMD three days following PDT.

\section{METHODS}

Three eyes of three patients presenting with neovascular AMD underwent CNV extraction three days after PDT. The patients had a complete ophthalmological examination including fundus photography and stereoscopic fluorescein angiography. Fluorescein angiography was performed before the PDT and three days later, on the day of surgery. All patients had subfoveal classic CNV. Visual acuity of the affected eyes varied between $4 / 200$ and 10/200. Since the visual acuity in all eyes was less than 20/200, they were not eligible for the PDT, according to inclusion criteria of the TAP-Investigation. ${ }^{11}$ The treatment options, including observation, conventional thermal laser photocoagulation, macular translocation with $360^{\circ}$ retinotomy and CNV extraction were advised with the patients. The patients were advised that PDT before macular surgery could reduce the bleeding of the lesion at the time of surgical extraction and the rate of recurrence. The experimental nature of the treatment procedure was discussed in detail and informed written consent was obtained. The study of the excised $\mathrm{CNV}$ specimens was approved by the institutional ethic committee.

Three days after PDT, the patients underwent macular surgery, consisting of a standard three port pars plana vitrectomy, retinotomy temporal to the macula, creation of a small macular detachment by injecting balanced salt solution through the retinotomy, removal of $\mathrm{CNV}$ using subretinal forceps, and a fluid-air exchange.

All specimens were fixed in 10\% neutrally buffered formalin, dehydrated, and embedded in paraffin for light microscopy. The membranes were serially sectioned and stained in a stepped fashion with Masson trichrome, periodic acid Schiff (PAS) and phosphotungstic acid haematoxylin (PTAH). The PAS staining was primarily used to confirm the location of the diffuse drusen and to help the overall orientation of the specimens whereas the PTAH stain showed the deposition of fibrin in the specimens.

\section{RESULTS}

\section{Case 1}

A 79 year old woman reported diminished vision in her right eye for three months. Visual acuity was 4/200 in the right eye. Ophthalmoscopic examination showed subretinal fluid and a subretinal greyish lesion subfoveally. Fluorescein angiography revealed subfoveal classic CNV (fig 1A). Three days after PDT, early phase of the fluorescein angiography showed nonperfusion of the irradiated area, including the CNV (fig 1B). The late phase revealed hyperfluorescence of the large choroidal vessels suggesting choroidal ischaemia and leakage

Abbreviations: $A M D$, age related macular degeneration; $C N V$, choroidal neovascularisation; PAS, periodic acid Schiff; PDT, photodynamic therapy; PTAH, phosphotungstic acid haematoxylin 

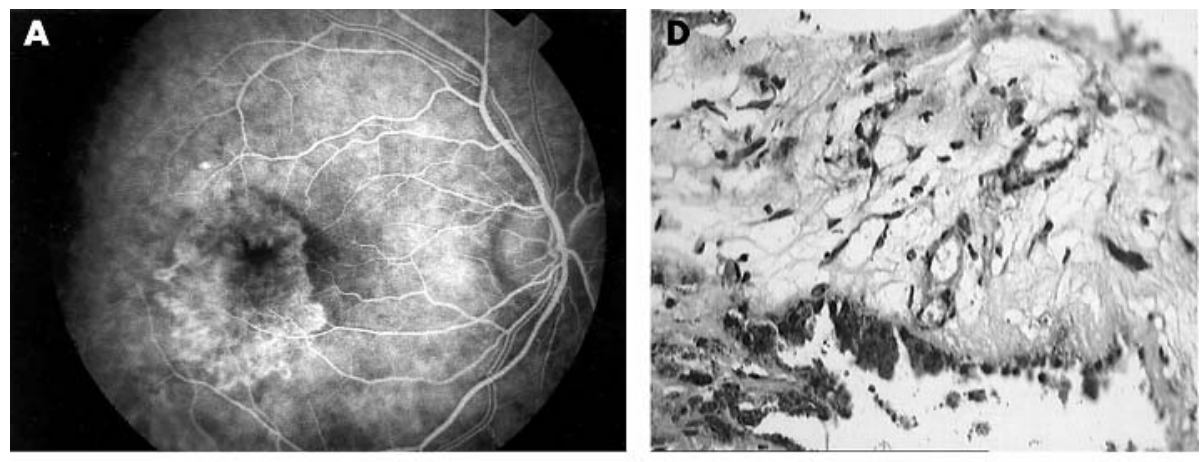

Figure 1 (A) Early phase fluorescein angiography shows a well defined bright hyperfluorescence consistent with subfoveal classic choroidal neovascularisation (CNV). (B) Three days after PDT, arterio-venous phase of the fluorescein angiography shows

non-perfusion the CNV and the treated area and staining of the large choroidal vessels at the margin of the treated area. (C) Late phase fluorescein angiography shows macular oedema. (D) Light micrograph, three days after PDT shows fibrovascular tissue (Masson trichrome; original magnification $\times 40$ ).
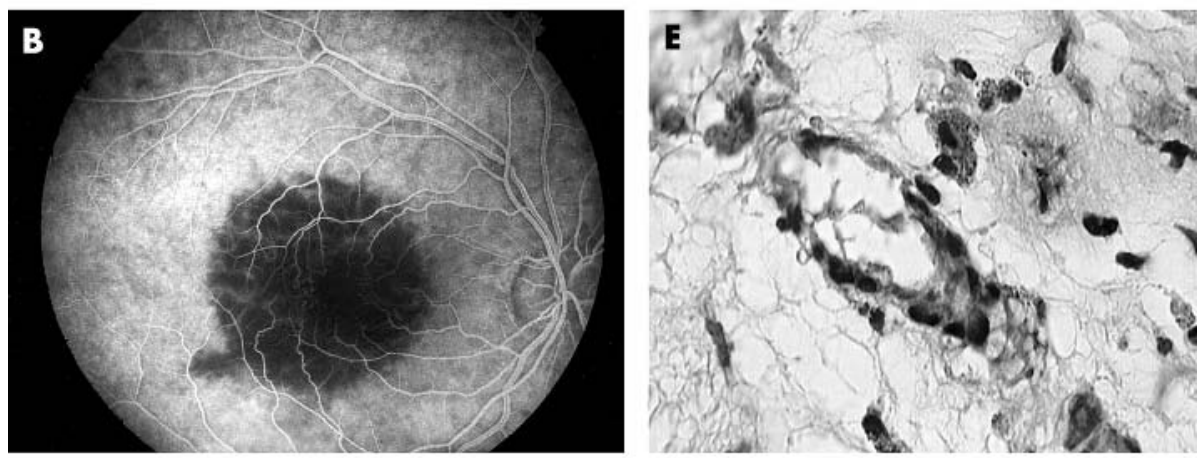

(E) Light micrograph shows a disintegrated vessel, probable and arteriole with swollen endothelium and some luminal debris without a thrombus in the subretinal fibrovascular tissue

(Masson trichrome; original magnification $\times 100$ ) (F) Some capillaries without a clear endothelial

lining and other vessels with some luminal debris (Masson trichrome; original magnification $\times 100$ ).
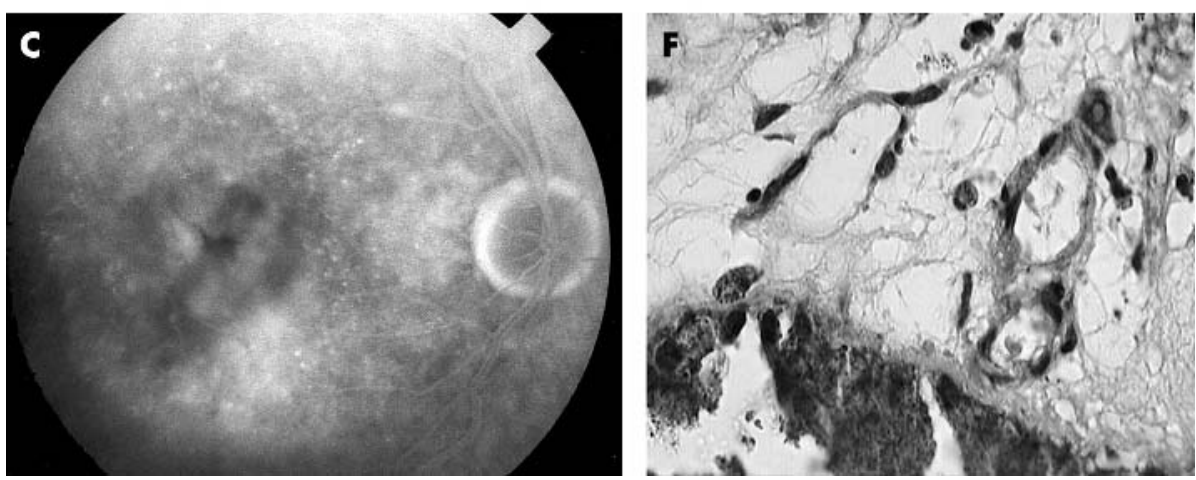

at the fovea (fig $1 \mathrm{C}$ ). The CNV was removed during uneventful macular surgery. On the first postoperative day, a small subretinal haemorrhage was seen under the macula, which resolved spontaneously. Six months later, the visual acuity was RE: 10/200. Ophthalmoscopic examination revealed the presence of subfoveal fibrosis.

\section{Histology}

The specimen contained retinal pigment epithelial cells and diffuse drusen which allowed the overall orientation of the specimen. It consisted of subretinal fibrovascular tissue which was relatively rich in cells but only a few capillaries and larger vascular structures were recognised. Their endothelium was swollen and/or disintegrated whereas their lumen did not appear to be occluded (fig lD-F).

\section{Case 2}

A 77 year old man was referred for exudative maculopathy in his left eye. He reported deterioration of his visual acuity over four months. His visual acuity was 5/200 in the left eye. Ophthalmoscopy revealed the presence of subretinal fluid and subretinal haemorrhages in the macular area. Fluorescein angiography was consistent with subfoveal classic CNV (fig 2A). Three days after the PDT, fluorescein angiography revealed non-perfusion of the treated area and CNV in the early phase as well as some leakage in the late phase (fig 2B). He underwent surgical removal of the CNV on the same day. No haemorrhage was observed during the surgery or in the early postoperative period. Three months later his visual acuity had remained 5/200. Ophthalmoscopy revealed a dry macula with atrophic changes foveally.

\section{Histology}

The specimen consisted of subretinal fibrovascular tissue bordered by retinal pigment epithelium and diffuse drusen. There was prominent fibrin exudation in the stroma which was moderately rich in cells but fibrin was not found in the vascular lumina (fig 2C-F). Several vascular elements, capillaries, and larger vessels with disintegrated endothelium were seen. The lumina of the CNV were not occluded and in some a marginal polymorph nuclear cell was recognised (fig 2D and F).

\section{Case 3}

An 83 year old man had blurry vision in his left eye for four months. The visual acuity in his left eye was 10/200. Ophthalmoscopy revealed the presence of subretinal fluid foveally and a subretinal haemorrhage superior to the fovea. Fluorescein angiography showed subfoveal classic CNV (fig 3A). Three days after PDT, the early phase of fluorescein angiography showed hypofluorescence at the macula (fig 3B). The late phases revealed leakage parafoveally. He underwent 

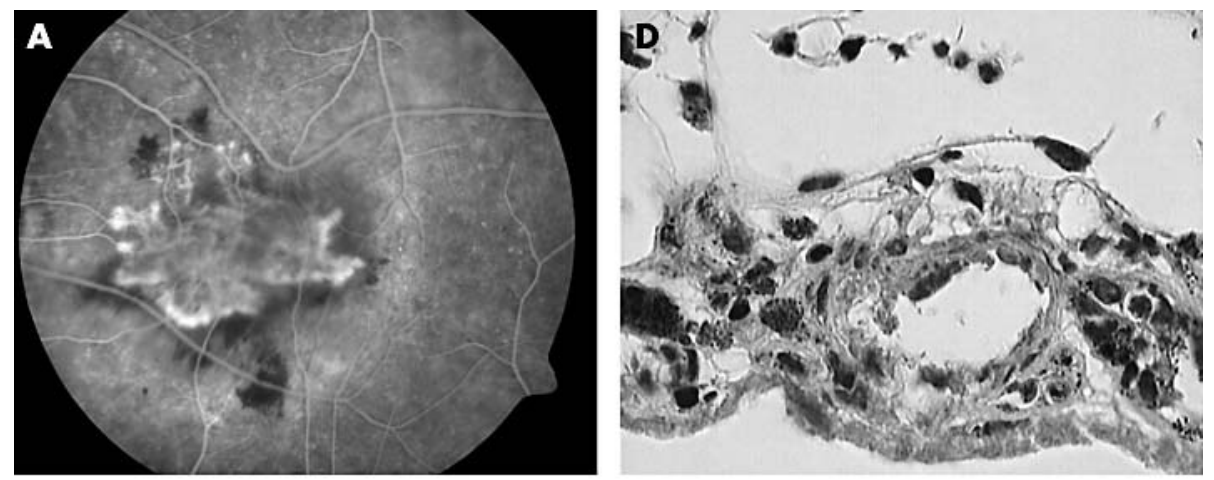

Figure 2 (A) Early phase fluorescein angiography reveals subfoveal classic CNV. (B) Three days after PDT, early phase fluorescein angiography shows non-perfusion of the CNV and the treated area at the macula. (C)

Overview of subretinally located fibrovascular tissue. Only a few vascular elements are retrieved even though the specimen is fairly rich in cells (Masson trichrome; original

magnification $\times 20$ ). (D) Detail of a capillary shows swollen endothelial nuclei and no occlusion of the lumen (Masson trichrome; original magnification $\times 100)$. $(E)$ and $(F)$ Fibrin deposition is found in the stroma of the
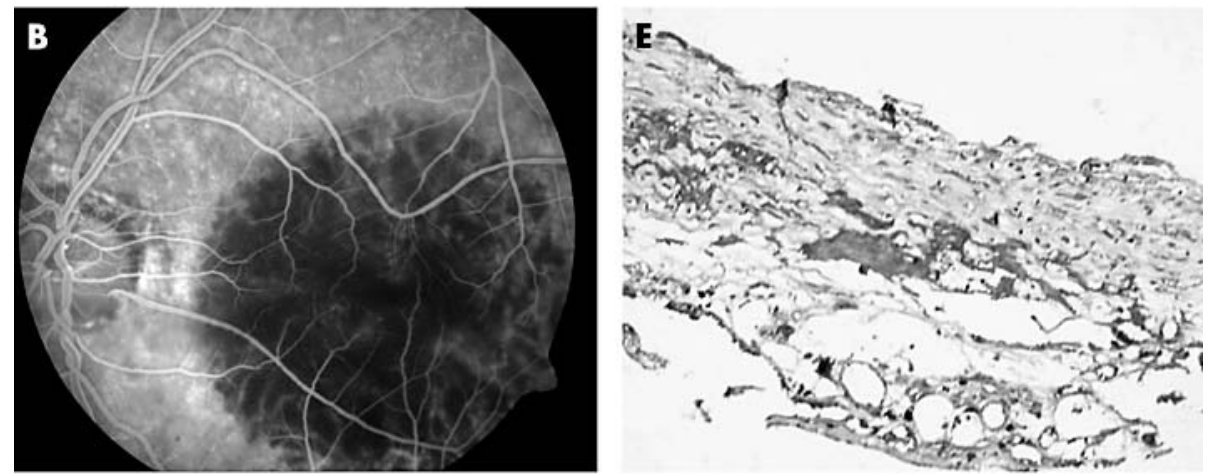

CNV but not within the vessels. The vascular endothelium is severely damaged and a marginal polymorph nuclear cell can be seen. Some luminal debris is found but an occlusion is not seen ((E) PTAH; original magnification $\times 40$ and (F) detail, PTAH; original magnification $\times 100$ ).
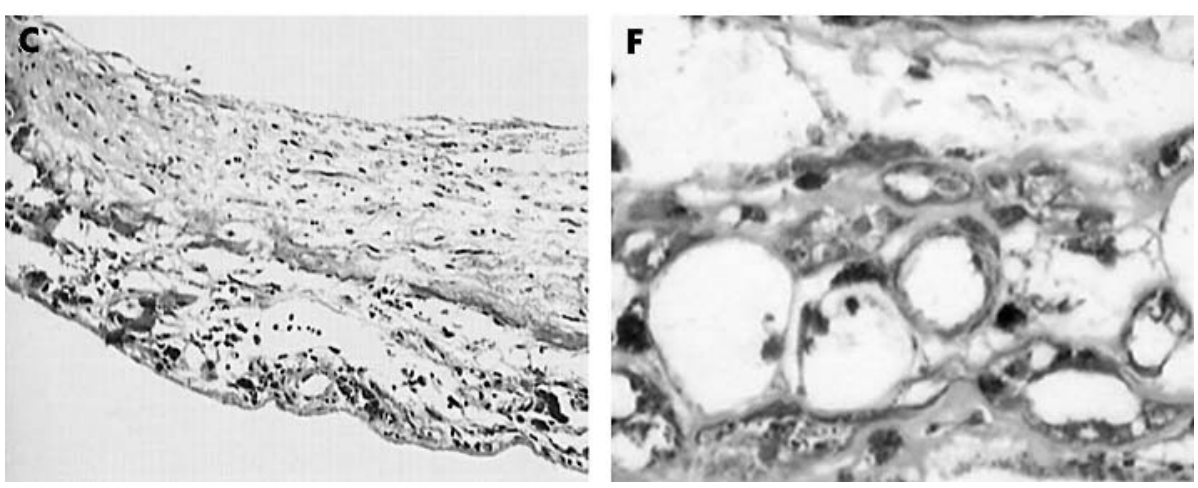

surgical removal of the CNV. The operation and the early postoperative course were uneventful. Six months later his visual acuity was 5/200 in the left eye. Ophthalmoscopy revealed a dry macula.

\section{Histology}

The specimens consisted of mixed subretinal and intraBruch's fibrovascular tissue; both of these components were separated by a conspicuous layer of diffuse drusen and retinal pigment epithelial cells. Several parts of the relatively large membrane were poor in cells and densely collagenised, whereas others were moderately cell rich. Capillaries were suspected in the subretinal as well as intra-Bruch's component whereas larger vessels only in the intra-Bruch's component. The endothelium of the vessels was either swollen or disintegrated. The lumina did not show an occlusion (fig 3C and D).

\section{DISCUSSION}

In this clinicopathological study, fluorescein angiography showed non-perfusion of the classic CNV and hypofluorescence of the entire irradiated area three days after PDT. Staining of some large choroidal vessels and late subfoveal leakage in the late phase of the fluorescein angiography was suggestive for some degree of choroidal ischaemia.
Histopathological examination of the excised CNV membranes revealed fibrovascular tissue with swollen and disintegrated endothelium; however neither a RBC nor a platelet/fibrin clot was observed. Fibrin deposition was seen in the stroma of the CNV on PTAH, a histochemical stain for fibrin, but fibrin was not found to be deposited in the vascular lumina.

Several histopathological studies of surgically excised CNV after PDT have been reported. Schnurrbusch et al, in an electron microscopic study of surgically extracted CNV, showed occluded vessels with thrombotic masses and ultrastructural damage of the neovascular endothelium three months after PDT. ${ }^{7}$ Ghazi et al also reported endothelial cell degeneration with platelet aggregation and thrombus formation on removed CNV specimens four weeks after PDT. ${ }^{8}$ They hypothesised that the clot fragmentation and re-endothelisation were responsible for repermeabilisation of the neovascular lesion. The central hyperfluorescence of the CNV could represent reperfused vessels following the initial occlusion after PDT. Grossniklaus et al studied a postmortem eye, after limited macular translocation, laser photocoagulation, and PDT. Complete occlusion of the persistent neovascularisation was shown histopathologically two weeks after the application of PDT. Platelet-fibrin thrombi were absent in the treated area. ${ }^{9}$ Moshfeghi et al reported on the histopathology of eight 

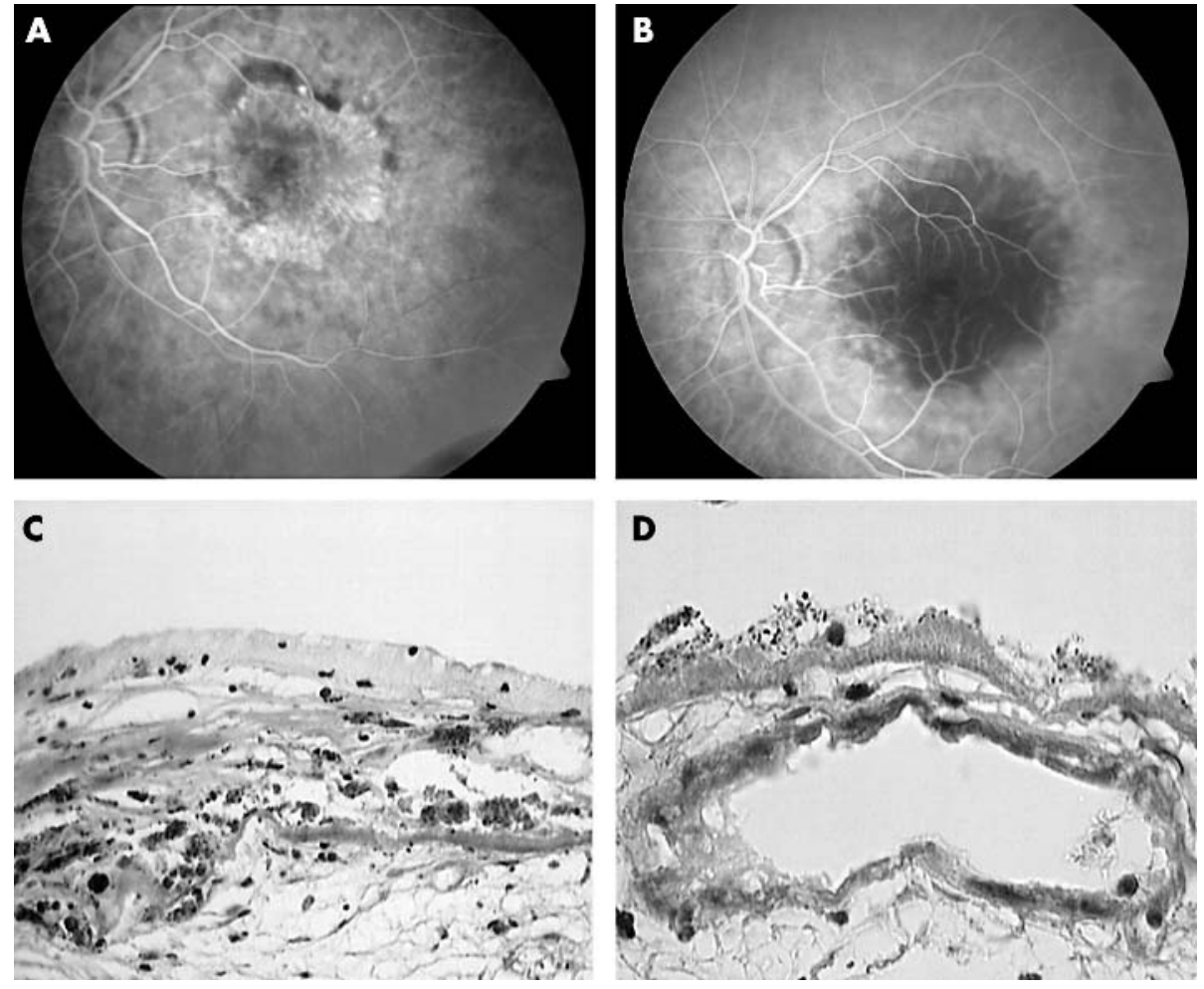

Figure 3 (A) Fluorescein angiography shows well defined early bright hyperfluorescence consistent with a subfoveal classic CNV. (B) Three days after PDT, early phase of the fluorescein angiography reveals hypofluorescence at the macula. (C) Detail showing subretinal and intra-Bruch's fibrovascular tissue separated by retinal pigment epithelium and diffuse drusen. A rare vascular element is suspected. Endothelial lining appears to be disintegrated (Masson trichrome; original magnification $\times 40$ ). (D) Detail of a vein lined by abnormal endothelium. A thrombus formation is not seen (Masson trichrome; original magnification $\times 100$ ).

surgically removed CNV membranes at different time intervals after PDT. $^{10}$ In his series, one CNV specimen secondary to presumed ocular histoplasmosis syndrome was removed three days after the second PDT. The time interval between the first and second PDT was one month. The patient had clinically active CNV and leakage shown by fluorescein angiography. Three days after the second PDT, fluorescein angiography revealed hypofluorescence in the treated area. Histopathologically, they found an area of vascular channels filled with erythrocytes and early thrombus formation, suggestive of vascular occlusion. They conclude that partial occlusion of the CNV could lead to subsequent vascular damage.

Our light microscopic findings of endothelial damage of CNV vessels three days after PDT confirm the previous histopathological observations. However, our study includes some new observations of surgically excised CNV membranes three days after PDT, such as the absence of a thrombus and occluded CNV vessels. Some differences between the reports make a comparison difficult. Except for the work of Moshfeghi et al, other reports are based on removed CNVs at later time intervals after PDT. Another limitation is the difference with regard to the number of the PDT sessions. Furthermore, the previously reported cases were either unresponsive or complicated-for example, haemorrhage following PDT.

Several studies revealed important features of CNV and the underlying choroid after PDT. Lin et al found platelet debris within the lumen of choroidal capillaries one hour after PDT in rabbit eyes. ${ }^{12}$ They also noted non-perfusion of the choroidal vasculature with gradual reperfusion beginning from the periphery three days following PDT. Light microscopy in this stage showed extravasated RBC in the choroid.
Schmidt-Erfurth et al have shown hypofluorescent halo surrounding the CNV shortly after PDT by using indocyanine green angiography, correlating with the applied treatment spot. ${ }^{13}$ The group of Schmidt-Erfurth also reported uniform occlusion of the healthy choriocapillaris one week following the PDT in enucleated human eyes. ${ }^{14}$ They concluded that the PDT at a dosage used clinically in the treatment of the AMD induced a selective destruction of vascular endothelial cells within the choriocapillary layer. In an experimental model, Flower et al showed reduced blood flow of the choriocapillaris affecting the perfusion of the CNV. ${ }^{15}$ We therefore suggest that the occlusion of the choriocapillaris to be the more likely explanation for the non-perfusion of $\mathrm{CNV}$ membranes in three days following verteporfin PDT in the absence of intraluminar thrombus and occlusion of the pathological new vessels, as seen in our study.

The selectivity of the verteporfin PDT and the temporary nature of its effect are important issues influencing the outcome of this treatment in neovascular AMD. Whether PDT induced ischaemia of the choriocapillaris influences the reactivation of the CNV by increasing the expression of factors contributing to new vessel formation is not yet known. Furthermore, how chronic choroidal hypoxias through multiple PDT sessions damage the retinal pigment epithelium and neurosensory retina has yet to be elucidated. In the current study, the absence of thrombosis in the CNV by light microscopic examination in the presence of fluorescein angiographically proven non-perfusion of the entire treated area, suggests choroidal ischaemia results in the closure of the CNV vessels in the early period following PDT. The detected disintegration of the endothelium of the CNV vessels may result in thrombosis in the weeks following treatment. The histopathological studies of 
PDT treated CNV may expand our knowledge on the mechanism of PDT.

\section{Authors' affiliations}

F Gelisken, W Inhoffen, M Voelker, S Grisanti, K U Bartz-Schmidt, Department of Ophthalmology I, University of Tuebingen, Germany B A Lafaut, Department of Ophthalmology, University of Ghent, Belgium Presented as a poster at the ARVO Meeting in Fort Lauderdale, USA, May 2002.

Correspondence to: Dr F Gelisken, Department of Ophthalmology I, University of Tuebingen, Schleichstr. 12, 72076 Tuebingen, Germany Faik.Gelisken@med.uni-tuebingen.de

Accepted for publication 7 June 2003

\section{REFERENCES}

1 Bird AC, Bressler NM, Bressler SB, et al. An international classification and grading system for age-related maculopathy and age-related macular degeneration. The International ARM Epidemiological Study Group. Surv Ophthalmol 1995;39:367-74.

2 Bressler NM, Bressler SB, Fine SL. Age-related macular degeneration. Surv Ophthalmol 1988:32:375-413.

3 Miller JW, Schmidt-Erfurth U, Sickenberg M, et al. Photodynamic therapy with Verteporfin for choroidal neovascularization caused by age-related macular degeneration: results of a single treatment in a phase 1 and 2 study. Arch Ophthalmol 1999;117:1161-73.

4 Treatment of age-related macular degeneration with photodynamic therapy (TAP) Study Group. Photodynamic therapy of subfoveal choroidal neovascularization in age-related macular degeneration with verteporfin: twoyear results of 2 randomized clinical trials-TAP report No. 2. Arch Ophthalmol 2001;119:198-207.

5 Verteporfin in photodynamic therapy study group. Verteporfin therapy of subfoveal choroidal neovascularization in age-related macular degeneration: two-year results of a randomized clinical trial including lesions with occult but no classic neovascularization-VIP report No. 2. Am J Ophthalmol 2001;131:541-60.

6 Schmidt-Erfurth U, Tayyaba H. Mechanisms of action of photodynamic therapy with verteporfin for the treatment of age-related macular degeneration. Surv Ophthalmol 2000;45:195-214.

7 Schnurrbusch UEK, Welt K, Horn LC, et al. Histological findings of surgically excised choroidal neovascular membranes after photodynamic therapy. Br J Ophthalmol 2001;85:1086-91.

8 Ghazi NG, Jabbour NM, De la Cruz ZC, et al. Clinicopathologic studies of age-related macular degeneration with classic subfoveal choroidal neovascularization treated with photodynamic therapy. Retina 2001;21:478-86.

9 Grossniklaus HE, Brooks HL, Sippy BD, et al. Retinal translocation and photodynamic therapy for age-related macular degeneration with classic choroidal neovascularization: a clinicopathologic case report. Retina 2002:22:818-24.

10 Moshfeghi DM, Kaiser PK, Grossniklaus HE, et al. Clinicopathologic study after submacular removal of choroidal neovascular membranes treated with verteporfin ocular photodynamic therapy. Am J Ophthalmol 2003; 135:343-50.

11 Treatment of age-related macular degeneration with photodynamic therapy (TAP) study group. Photodynamic therapy of subfoveal choroidal neovascularization in age-related macular degeneration with verteporfin. One-year results of 2 randomized clinical trials. TAP Report 1. Arch Ophthalmol 1999;117:1329-45.

12 Lin SC, Lin CP, Feld JR et al. The photodynamic occlusion of choroidal vessels using benzoporphyrin derivative. Curr Eye Res 1994:13:513-22.

13 Schmidt-Erfurth U, Michels S, Irene Barbazetto, et al. Photodynamic effects on choroidal neovascularization and physiological choroid. Invest Ophthalmol Vis Sci 2002;43:830-41.

14 Schmidt-Erfurth U, Laqua H, Schloetzer-Schrehard U, et al. Histopathological changes following photodynamic therapy in human eyes. Arch Ophthalmol 2002;120:835-43.

15 Flower RW, von Kerczek C, Zhu L, et al. Theoretical investigation of the role of choriocapillaris blood flow in treatment of subfoveal neovascularization associates with age-related macular degeneration. Am J Ophthalmol $2001 ; 132: 85-93$.

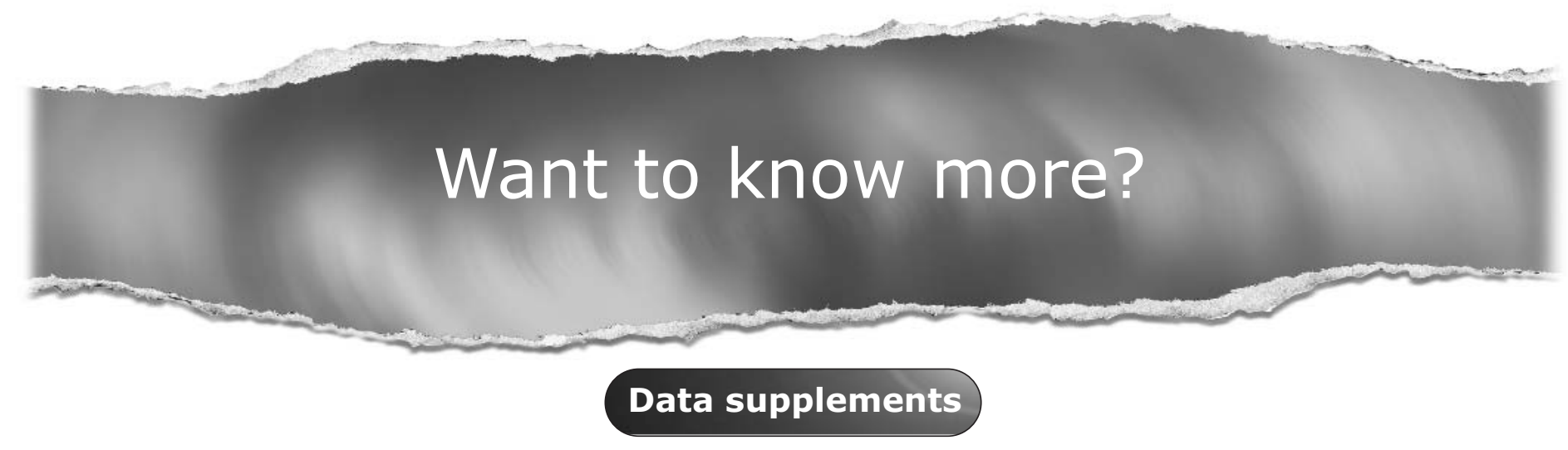

Limited space in printed journals means that interesting data and other material are often edited out of articles; however, limitless cyberspace means that we can include this information online. Look out for additional tables, references, illustrations, and (soon to appear) multimedia clips online!

\section{www.bjophthalmol.com}

